\title{
Estudios de internet, comunicación y cultura digital en México
}

\section{Internet, digital communication and culture studies in Mexico}

Dorismilda Flores-Márquez ${ }^{1}$

Resumen: Este artículo presenta una revisión de publicaciones sobre internet, comunicación y cultura digital, en México, durante el periodo 1981-2019, con el fin de identificar diferentes etapas, así como la presencia de rastros del pensamiento comunicacional latinoamericano y sus líneas derivadas. Para ello, se realizó una revisión de productos en CCDoc, una base de datos especializada en el campo de la comunicación en México. Se registraron 725 textos -libros, capítulos y artículos-, se hizo un análisis de contenido y, posteriormente, una línea de tiempo, para identificar las principales temáticas e investigadores clave, las etapas en la investigación y los vínculos con el pensamiento comunicacional latinoamericano y otras tradiciones. Estos hallazgos desafían a otras generaciones de investigadores a reconocer las herencias y a contribuir al campo.

Palavras-Chave: Estudios de internet; cultura digital; comunicación digital; medios digitales; México.

Abstract: This article presents a review of Mexican publications on internet, digital communication and culture, during the period 1981-2019, in order to identify different stages, and the presence of traces of the Latin American communication thinking and its branches as well. For that purpose, a review of academic products was conducted, in CCDoc, a database specialized in the Mexican communication field. 725 texts - among books, chapters and articles - were

1 Universidad De La Salle Bajío (ULSAB). León, México.

https://orcid.org/0000-0002-9659-6392 E-mail: dfloresm@delasalle.edu.mx 
registered, a content analysis was carried out and a timeline was made, to identify the main topics and key researchers, the stages of the research, and the links with the Latin American communication thinking and with other traditions as well. These findings open a challenge to newer generations of researchers, to recognize their heritage and to contribute to the field.

Keywords: Internet studies; digital culture; digital communication; digital media; Mexico. 


\title{
Presentación
}

\author{
La pregunta de partida es por \\ cuánto país cabe en los discursos de la academia, \\ los medios de comunicación y la historia que se escribe, \\ pero apuntando a un relato de futuro. \\ Jesús Martín-Barbero
}

Los estudios sobre internet, comunicación y cultura digital configuran un campo de estudio joven e interdisciplinario: joven, porque se concentra principalmente en las tres décadas más recientes; interdisciplinario, en tanto que la complejidad de los objetos ha sido abordada a partir de distintos campos académicos, e incluso de otros campos del saber y la vida social, como el periodismo y la política (FUENTES NAVARRO, 2000; SCOLARI, 2008; SILES, 2008; TREJO DELARBRE, 1996).

Si bien en los años recientes ha crecido y se ha diversificado el interés por el estudio de lo digital, la pandemia de la COVID-19 en 2020 marca un momento clave. Los periodos de cuarentena en distintos países implicaron el repliegue a los hogares y el traslado de tareas a entornos digitales, en muchos casos, sin las condiciones adecuadas para ello. Esto se tradujo en desafíos y oportunidades y tuvo su correlato en el aumento de estudios sobre la digitalización en tiempos de pandemia. Es pertinente, entonces, hacer un balance del trabajo realizado hasta el momento, que sirva como guía y permita identificar los cambios.

Por otro lado, la presencia de internet y los medios digitales a nivel global permite encontrar elementos comunes, a la vez que se evidencian las desigualdades y particularidades de apropiación. Si bien este campo de estudio se desarrolla en todo el mundo, hay una predominancia de referentes anglo-europeos, que a veces se asumen acríticamente. Conviene revisar el desarrollo de investigaciones en diferentes regiones, para identificar sus lógicas y contribuciones en relación con el contexto, para reconocerlas y diversificar la discusión.

En Latinoamérica hay un intenso desarrollo de los estudios de comunicación, pero su difusión se concentra en la propia región y no 
necesariamente gana visibilidad fuera de ella (ENGHEL \& BECERRA, 2018; GANTHER \& ORTEGA, 2019). Esta condición se vive también en los estudios sobre cultura digital (GÓMEZ-CRUZ \& SILES, 2020).

Las revisiones sobre los estudios latinoamericanos de internet, comunicación y cultura digital han reconocido el crecimiento y las aportaciones, pero también han señalado la necesidad de teorización, desarrollo metodológico, así como perspectivas regionales y comparativas (FUENTES NAVARRO, 2000, 2011; GÓMEZ-CRUZ \& SILES, 2020; SCOLARI, 2008; SILES, 2008; SILES, ESPINOZA ROJAS, \& MÉNDEZ MARENCO , 2019; TREJO DELARBRE, 1996, 2009, 2013).

El caso mexicano presenta lógicas similares a las latinoamericanas. Por un lado, se aprecia el crecimiento del interés y, por otro, la falta de desarrollo teórico y metodológico, a la cual se suma una tendencia a citar los autores clásicos más que a los contemporáneos (MENESES \& PÉREZ SALAZAR, 2016; TREJO DELARBRE \& SOSA PLATA, 2009). Esto contrasta con el desarrollo de lo que algunos autores han llamado pensamiento comunicacional latinoamericano (MARQUES DE MELO, 2011).

Este artículo presenta una revisión de publicaciones sobre internet, comunicación y cultura digital, en México, orientada a identificar relaciones y aportaciones al pensamiento comunicacional latinoamericano. La delimitación en México se sostiene sobre tres razones principales. De entrada, fue - junto con Brasil - uno de los primeros países latinoamericanos a los que llegó internet a principios de la década de 1990. En segundo lugar, uno de los casos más emblemáticos del uso de internet ha sido el movimiento zapatista, que se levantó en 1994 en la selva del sureste mexicano y cuya presencia en la red fue un hito. En tercer lugar, uno de los dos primeros libros publicados en Latinoamérica sobre el tema fue La nueva alfombra mágica: Usos y 
mitos de internet, la red de redes del mexicano Raúl Trejo Delarbre². Esto configura un escenario que vale la pena analizar.

La revisión incluyó 725 textos - libros, capítulos y artículos -, publicados entre 1981 y 2019, en torno a nuevas tecnologías, internet, medios digitales, comunicación digital y cultura digital. Se realizó un análisis de contenido cuyos resultados se tradujeron en una línea de tiempo, como puede verse en las notas metodológicas.

Como se verá en las siguientes secciones, a lo largo de los años se aprecia una transición entre los abordajes tempranos sobre la incorporación de las TIC, la búsqueda de comprender y proveer de materiales teóricos y metodológicos, así como la orientación hacia los trabajos empíricos. También es posible observar rasgos del pensamiento comunicacional latinoamericano y sus derivados, así como de otras perspectivas, como el pensamiento de McLuhan y el pensamiento complejo. La revisión abre desafíos para el futuro de los estudios sobre internet, comunicación y cultura digital en México y Latinoamérica.

\section{El pensamiento comunicacional como encuadre}

Entre las opciones posibles para analizar el desarrollo los estudios sobre internet, comunicación y cultura digital, he optado por una serie de delimitaciones. En primer lugar, no se abordan los estudios de internet en general, sino aquellos hechos desde marcos comunicacionales. Por el carácter interdisciplinario de los objetos y del propio campo, se encontrarán intersecciones con otras líneas y preocupaciones, pero el foco del trabajo es comunicacional.

La perspectiva desde la cual se mira es el pensamiento comunicacional latinoamericano, lo que implica identificar, con cierto nivel de claridad, una tradición o un conjunto de tradiciones que comparten determinadas características. Se asume que éstas se construyen en torno al campo de la comunicación, en una región específica, que es Latinoamérica.

2 El otro es Ciberculturas en la era de las máquinas inteligentes, del argentino Alejandro Piscitelli. 
De acuerdo con Miège (1995), el pensamiento comunicacional no se reduce a la producción científica, sino que incluye otros campos - como el profesional - y las interrogantes de las sociedades. En este sentido, el autor señala tres aspectos: 1) "su estrecho vínculo con acciones sociales (estrategias públicas y privadas, habilidades profesionales, comportamientos de usuarios-consumidores...), y cuestiones procedentes de lugares especializados en la mediación cultural y la transmisión de conocimientos"; 2) la transversalidad entre diferentes campos; 3 ) la "facultad de integrar problemáticas provenientes de corrientes teóricas diferentes” (p. 110).

En su propuesta, el pensamiento comunicacional se ha construido académicamente a partir de las corrientes fundadoras, la expansión de las problemáticas y las interrogantes presentes. Éstas corresponden a etapas identificables en el tiempo, a partir de la década de 1930. Se identifican de este modo las preocupaciones traducidas en objetos de estudio, teorías y metodologías, que son propias de una época y/o una región y hablan de contextos diferenciados, así como las contribuciones que desde ahí se hacen al campo de la comunicación.

La presencia de Latinoamérica en el mapa de los estudios de comunicación implica tensiones entre la importación de modelos anglo-europeos y las producciones propias, contextualizadas, críticas, que algunos interpretamos como una especie de resistencia creativa, aunque no siempre visible fuera del continente. En este sentido, José Marques de Melo (2011), un académico brasileño que abordó el pensamiento comunicacional latinoamericano, planteó que éste "se distingue de sus matrices referenciales, europeas o norteamericanas, por el compromiso que asume en relación al bien común, o sea, al interés público y a las aspiraciones colectivas" (p. 10). Él identificó, principalmente en los trabajos seminales del campo, cierto énfasis en los derechos humanos y la intervención sobre la realidad social.

De acuerdo con Raúl Fuentes Navarro (2011), la idea del pensamiento comunicacional sirve "para reconstruir una historia, en la que ciertos aportes se consideran más valiosos o significativos que otros, para 
justificar las perspectivas adoptadas en el presente, y para desde ahí trazar líneas de desarrollo y acción futuras" (p. 44). El autor agrega que "la investigación de la comunicación en América Latina puede muy bien revisarse, en sus trayectorias pasadas y en vistas al futuro, como una larga serie de retos tanto internos (científicos, académicos) como sobre todo externos (socioculturales, políticos)" (p. 48).

Por otra parte, Gabriel Kaplún (2013) ha identificado cuatro tradiciones principales en el campo de la comunicación en Latinoamérica: funcionalista, crítica, culturalista y alternativista. La primera de ellas no necesariamente tuvo su propio desarrollo en este continente, sino que importó propuestas teóricas para el análisis de las funciones de los medios. La tradición crítica emancipatoria, basada en la escuela de Frankfurt y los estudios semióticos, se ha enfocado en el poder y las estructuras de los medios. Entre sus figuras clave están Armand Mattelart - académico belga, que radicó por muchos años en Chile- y Héctor Schmucler - académico argentino, que pasó varios años exiliado en México.

Las tradiciones culturalista y alternativista coinciden en varios elementos, pero difieren en otros, una está ligada a la academia y otra a la intervención social. En la culturalista se ha abordado la presencia de lo popular en la comunicación y la cultura, principalmente a partir del trabajo de dos autores clave: Jesús Martín-Barbero - filósofo español, que ha pasado la mayor parte de su vida en Colombia y vivió algunos años en México - y Néstor García Canclini - antropólogo argentino radicado en México. Finalmente, en la tradición alternativista el autor agrupa a distintas denominaciones, como la comunicación popular, la comunicación alternativa y otras. Con el fin de abrir alternativas frente a los medios masivos, esta tradición se enfocó al trabajo en organizaciones no gubernamentales de educación popular, emisoras locales de radio y centros culturales comunitarios. Se configuró más como un campo de intervención que de producción teórica, en el que han convivido activistas, comunicadores profesionales y académicos (KAPLÚN, 2013). 
Estas tradiciones principales del pensamiento comunicacional latinoamericano se han diversificado a lo largo de los años, como puede verse en las publicaciones, los congresos, así como los programas de formación en universidades y de investigación. Esta diversidad puede verse en las asociaciones de investigadores, como la Asociación Latinoamericana de Investigadores de la Comunicación (ALAIC) $)^{3}$ o la Asociación Mexicana de Investigadores de la Comunicación (AMIC) $)^{4}$. En ellas se identifican tanto las raíces que dieron origen a las discusiones, como la fragmentación y la desarticulación que caracteriza al campo académico de la comunicación (FUENTES NAVARRO, 2011). Así, este trabajo orienta la revisión de estudios sobre internet, comunicación y cultura digital, hacia la identificación de etapas en la investigación y rasgos de tradiciones del pensamiento comunicacional latinoamericano.

\section{Notas metodológicas}

Todo recorte es problemático. Delimitar la búsqueda a México abre otras preguntas: ¿se trata de investigaciones hechas por mexicanos?, ¿investigaciones hechas en México? Estas preguntas abren otras reflexiones sobre cómo ubicar, por ejemplo, a investigadores mexicanos que ahora residen en otros países ${ }^{5}$ o a investigadores extranjeros, que han vivido en

3 ALAIC tiene 19 grupos temáticos: 1) comunicación intercultural y folkcomunicación, 2) comunicación organizacional y relaciones públicas, 3) comunicación política y medios, 4) comunicación y educación, 5) comunicación y salud, 6) economía política de las comunicaciones, 7) estudios de recepción, 8) comunicación popular, comunitaria y ciudadanía, 9) teoría y metodología de la investigación en comunicación, 10) comunicación, tecnología y desarrollo, 11) comunicación y estudios socioculturales, 12) comunicación para el cambio social, 13) comunicación comunitaria, 14) discurso y comunicación, 15) comunicación y ciudad, 16) estudios sobre periodismo, 17) comunicación e historia, 18) ética, libertad de expresión y derecho a la comunicación, 19) comunicación digital, redes y procesos.

4 AMIC tiene 16 grupos de investigación: 1) nuevas tecnologías, internet y sociedad de la información, 2) comunicación política, 3) economía política de la comunicación, 4) estudios de recepción, 5) estudios de periodismo, 6) comunicación y educación, 7) discurso, semiótica y lenguaje, 8) comunicación integral en las organizaciones, 9) historia de la comunicación, 10) teorías y metodología de la investigación en comunicación, 11) comunicación intercultural, 12) género y comunicación, 13) sociedad civil, participación y comunicación alternativa, 14) políticas de la comunicación, 15) comunicación intersubjetiva, 16) estudios de juventud y comunicación; además de cuatro grupos de trabajo: 1) deporte, cultura y sociedad, 2) medios públicos, 3) comunicación de la ciencia, 4) comunicación y salud.

5 Tales son los casos de Edgar Gómez Cruz y Octavio Islas. 
México y han producido parte de su obra en este país ${ }^{6}$. En ambos casos se tomaron los trabajos realizados por ellos mientras estaban en México.

La revisión se concentró en CC-Doc ${ }^{7}$, una base de datos de acceso abierto, especializada en comunicación, originada en la sistematización documental de productos de investigación sobre comunicación en México, encabezada por Raúl Fuentes Navarro. El proyecto articula el interés práctico por llevar un registro de la producción científica con el ideal de "contribuir a contrarrestar la fragmentación que afecta al campo” (FUENTES NAVARRO, 2014).

Si bien se trata de una base de datos muy amplia, los productos académicos registrados en ella no necesariamente son todos los que existen. También se asume que las palabras clave que etiquetan estos textos en la plataforma pueden tener limitaciones, sobre todo en el caso de trabajos que tocan distintas áreas y quedan registrados en líneas diferentes a las consultadas. Sin embargo, se trata de un esfuerzo de documentación muy complejo que debe ser reconocido, por su especialización en el campo de la comunicación, su longevidad - el proyecto fue pionero y ha operado desde el año 2000 - y su capacidad de integración incluye productos académicos, como libros y capítulos, que no están contemplados en otras bases de datos centradas en revistas científicas.

En CC-Doc se realizaron varias búsquedas, con los siguientes términos clave: nuevas tecnologías, internet, medios digitales, comunicación digital y cultura digital. No se hizo recorte temporal, sino que se revisaron todos los productos registrados bajo estos términos. Para la selección, se tomaron en cuenta libros, capítulos en libros coordinados, así como artículos publicados en revistas indexadas, no indexadas y de divulgación. Se asume que en estas publicaciones se materializan los avances y resultados de investigación y permiten dar cuenta de las líneas de estudio y sus cambios a través del tiempo. La base de datos incluye fichas

6 Tales son los casos de Héctor Schmucler, Jesús Martín Barbero, Cristóbal Cobo y Emiliano Treré.

7 Disponible en: https://ccdoc.iteso.mx/ 
de todas las publicaciones ${ }^{8}$ y en buena parte de ellas se incluye el texto completo. Se recuperaron tanto las fichas como los documentos disponibles. Se eliminaron registros duplicados, así como aquellos que no correspondían a los temas planteados en esta exploración.

Luego de la depuración, se consideraron 725 productos académicos, publicados entre 1981 y 2019. Con los registros se realizó un análisis de contenido (KRIPPENDORFF, 2004). Para ello se elaboró una base de datos, que incluyó año, título, autor/a/es, tipo de producto, tema, teoría y metodología. Esto permitió identificar temáticas, objetos de estudio, teorías y metodologías, así como autores clave.

Con esos datos se elaboró una línea de tiempo que, en la primera fase, a la que corresponde el presente artículo, se enfoca en las temáticas emergentes y los autores clave. Las temáticas emergentes son aquellas que representan una novedad en el campo, respecto a las tendencias identificadas en las publicaciones previas. Estas temáticas se enmarcan teórica y metodológicamente y eso permite asociarlas con tradiciones de investigación que, para efectos de este trabajo, corresponden (o no) a las tradiciones del pensamiento comunicacional latinoamericano (KAPLÚN, 2013; MARQUES DE MELO, 2011). Los autores clave son aquellos que introducen las temáticas emergentes en el campo y/o logran mantenerse en estas líneas de investigación durante varios años, lo cual contribuye a la consolidación del campo.

\section{Panorama de estudios sobre internet, comunicación y cultura digital en México}

La revisión incluyó 725 textos, entre libros, capítulos de libros y artículos en revistas, que fueron publicados entre 1981 y 2019. La distribución por décadas puede observarse en la tabla 1. Hay un crecimiento en el

8 Ésta incluye número de registro, título, autor, editor, fecha, tipo, formato, descripción, materia, fuente y anexos. 
volumen de publicaciones en las primeras tres décadas y cierta estabilización en la última.

Tabla 1 - Productos analizados, por décadas

\begin{tabular}{|l|l|l|l|l|}
\hline Periodo & $1980-1989$ & $1990-1999$ & $2000-2009$ & $2010-2019$ \\
\hline Libros & 9 & 11 & 24 & 20 \\
\hline Capítulos & 17 & 26 & 130 & 102 \\
\hline Artículos & 26 & 63 & 138 & 159 \\
\hline Total de productos & 52 & 100 & 292 & 281 \\
\hline
\end{tabular}

Fuente: Elaboración propia

Por otro lado, se aprecia una clara predominancia de algunas temáticas que, desde distintas perspectivas teóricas y metodológicas, se abordado en estos casi 40 años. Las y los investigadores mexicanos han puesto mucha atención a objetos de estudio sobre educación, sociedad de la información y juventudes, entre otros que, en cierto sentido, son un reflejo del contexto y las problemáticas nacionales.

Tabla 2 - Temáticas más recurrentes

\begin{tabular}{|l|c|}
\hline \multicolumn{1}{|c|}{ Tema } & Periodo \\
\hline Educación & 83 \\
\hline Sociedad de la Información & 39 \\
\hline Juventudes & 38 \\
\hline Periodismo digital & 33 \\
\hline Movimientos sociales & 31 \\
\hline Internet / medios digitales & 24 \\
\hline Comunicación política & 20 \\
\hline Brecha digital / inclusión digital & 19 \\
\hline Políticas y regulación & 18 \\
\hline Televisión y convergencia & 15 \\
\hline
\end{tabular}

Fuente: Elaboración propia 
Más allá de las cantidades, pensar esta revisión como una línea de tiempo permite observar algunos elementos importantes. De entrada, como habían registrado varios autores, hay un interés temprano por el tema en México (FUENTES NAVARRO, 2000; TREJO DELARBRE, 1996). Si bien los textos que abordan de manera explícita a internet se sitúan en la década de 1990, desde la previa hay textos sobre revoluación tecnológica, satélites, computadoras, tecnologías de información y comunicación - en algunos casos aparecen todavía como nuevas tecnologías de información y comunicación, en otros como tecnologías de información y comunicación y en otros como tecnologías de comunicación-, de modo general, o bien en relación con la educación, el poder, el periodismo y/o la cultura.

Un ejemplo son los dos textos más antiguos que se encontraron en esta revisión. El primero fue "La hora de las computadoras. El estudio de la comunicación en América Latina”, publicado por Héctor Schmucler en la revista Connotaciones. En él se abordan las dos corrientes que el autor identificaba en el estudio de la comunicación - el desarrollo y la dependencia - en relación con los desafíos que planteaba la incorporación de tecnologías: "Se abre para la investigación sobre comunicación en América Latina un amplio camino para recorrer que encierra un desafío histórico: anticiparse a los acontecimientos y no resignarse a estudiar, retroactivamente, los efectos de los nuevos sistemas comunicativos" (SCHMUCLER, 1981, P. 61). El autor, por cierto, es reconocido como figura clave del pensamiento comunicacional latinoamericano de orientación crítica (KAPLÚN, 2013).

El segundo texto fue el libro Las empresas transnacionales en el campo de las comunicaciones internacionales vía satélite: COMSAT e INTELSAT. Cronología selecta de 1962 a 1980, de Jesús Roldán Acosta, publicado en la UNAM en 1981. No es un trabajo eminentemente comunicacional, pero aporta elementos para comprender las preocupaciones de la época y la orientación crítica desde la que se leían los cambios tecnológicos - que para entonces llevaban varias décadas - en el campo. 
En ese periodo se registró una preocupación por el vínculo entre TIC y poder, entre autores como Javier Esteinou, Fátima Fernández Christlieb y Florence Toussaint; así como por los usos socioculturales y educativos de las tecnologías, entre otros autores, como Raúl Fuentes Navarro, Delia Crovi Druetta y Carmen Gómez Mont.

La siguiente década, de 1990 a 1999, se caracterizó por la llegada de internet a las discusiones y la apertura de preguntas y líneas. Un momento clave fue la publicación del libro La nueva alfombra mágica. Usos y mitos de Internet, la red de redes, de Raúl Trejo Delarbre, con FUNDESCO y Editorial Diana. En éste, el autor presenta una visión panorámica sobre la red y sus diferentes dimensiones e implicaciones en la vida cotidiana. Se trata de un libro considerado seminal en las discusiones sobre internet en Latinoamérica.

Además, hubo una fuerte concentración en el vínculo entre TIC y educación, así como TIC y sus implicaciones para los medios - la prensa, la radio y la televisión. Asimismo, emergieron algunos temas clave, como el movimiento zapatista en internet, realidad virtual, videojuegos, cibercultura y el vínculo entre TIC y mujeres. Continuaron presentes algunos autores como Carmen Gómez Mont, Fátima Fernández Christlieb y Raúl Fuentes Navarro; y emergieron otros como Raúl Trejo Delarbre, Claudia Benassini Félix, Octavio Islas, Fernando Gutiérrez y Jesús Galindo. Algunos de ellos ya tenían trayectoria en el estudio de la comunicación y la sociología.

La siguente década, de 2000 a 2009 se caracterizó por la explosión del interés en el tema. Crecieron las discusiones sobre la sociedad de la información y las implicaciones de las TIC en diferentes áreas de la vida social. Se posicionaron nuevas discusiones sobre comunidades virtuales, ciberespacio y cibercultura, así como acerca de la relación entre movimientos sociales e internet. También creció el abordaje de los usos de las TIC y emergió el interés por las narrativas y la visualidad, así como por las prácticas comunicativas en plataformas específicas, tales como los blogs. 
Algunos textos que se orientaron a plantear fundamentos teóricos y metodológicos, tales como los libros Viviendo en el Aleph. La sociedad de la información y sus laberintos de Raúl Trejo Delarbre y Lectores, espectadores e internautas de Néstor García Canclini, publicados en 2006 y 2007 respectivamente.

También los siguientes textos: "Exploraciones teórico-metodológicas para la investigación sociocultural de los usos de Internet" de Raúl Fuentes Navarro, publicado en 2001; "Los usos sociales de las tecnologías de información y comunicación. Fundamentos teóricos” de Carmen Gómez Mont, "Tecnicidades, identidades, alteridades: des-ubicaciones y opacidades de la comunicación en el nuevo siglo" de Jesús Martín-Barbero, "Mediaciones tecnológicas y des-ordenamientos comunicacionales" de Guillermo Orozco Gómez y "Hacia la construcción de una metodología para el estudio de las comunidades virtuales" de Edgar Gómez Cruz, publicados en 2002; así como "Comunicología y sociedad de la información: Exploración de un lugar común desde la ciencia de la comunicación” de Jesús Galindo Cáceres, publicado en 2009.

Hubo, además, balances como "La investigación latinoamericana sobre Internet. Brechas digitales y diversidad analítica” de Raúl Trejo Delarbre, en 2004; “Teorías e investigación de Internet en México” de Octavio Islas y Fernando Gutiérrez, en 2005; así como "Campo nuevo, problemas viejos. La investigación mexicana en materia de Sociedad de la Información, Internet, Cibercultura y Telecomunicaciones", de Raúl Trejo Delarbre y Gabriel Sosa Plata, en 2009.

Un elemento importante de esta década fue la tendencia a publicar cada vez más textos con resultados de trabajos empíricos. Asimismo, se registró un vínculo con los debates políticos de la época, en términos de regulaciones, políticas públicas, así como del lugar que ocuparía internet en la vida política y social.

En la década más reciente, de 2010 a 2019, se sostuvo el abordaje de internet en relación con educación y política. También se registró un buen número de investigaciones sobre el uso de internet y redes sociodigitales en campañas electorales, principalmente en torno a las 
elecciones presidenciales en México en 2012 y 2018. De igual manera, crecieron los estudios sobre movimientos sociales e internet, a raíz de la emergencia de \#YoSoyl 32 en 2012 y las movilizaciones por Ayotzinapa. Si bien el tema ya estaba presente en las décadas anteriores, en torno al movimiento zapatista, en este periodo hubo un viraje hacia los movimientos en red, por parte de autores como Emiliano Treré y Guiomar Rovira, entre otros.

Se identificó una tendencia a centrarse en plataformas específicas, como Facebook, Twitter o YouTube. Se incorporó también un cuerpo de estudios sobre memes, en relación con acontecimientos políticos. En la línea de internet y educación se sostuvo el interés por las alfabetizaciones mediática, informacional y digital, así como por las competencias digitales.

Entre las aportaciones teóricas y metodológicas del periodo, se contemplan el libro Internet como medio de comunicación. Teoría y análisis de actos comunicativos en los entornos virtuales de Gabriel Pérez Salazar y “Apuntes para el análisis de prácticas de comunicación dialógica en mundos virtuales" de Magdalena López de Anda, ambos publicados en 2012; así como Cibercultur@e iniciación en la investigación interdisciplinaria de Margarita Maass Moreno, José Amozurrutia y Jorge González y "Cultura algorítmica y tecnologías del lenguaje humano" de Jesús Octavio Elizondo y Esaú Villatoro, ambos publicados en 2015.

En el período hubo revisiones importantes del estado de la investigación sobre estos temas. Tal es el caso del libro Sociedad del conocimiento y comunicación: Reflexiones críticas desde América Latina, coordinado por Delia Crovi Druetta en 2018; “Tras los hilos que tejen la red: Una aproximación a la investigación sobre Internet en las revistas de comunicación en América Latina” de José Manuel Corona y Gabriela Gómez Rodríguez, publicada en 2010; años después, en 2016, fue publicada "Cómo y qué se estudia sobre Internet y la Sociedad de la Información en México. Una mirada desde la AMIC” de María Elena Meneses y Gabriel Pérez Salazar. 
Algunos textos dejan evidencia de la participación en proyectos transnacionales. Tal es el caso de "Principales indicadores de Internet en la sociedad mexicana" de Octavio Islas y Amaia Arribas, publicado en 2010, a partir del World Internet Project; Mapping digital media Mexico, de Rodrigo Gómez y Gabriel Sosa Plata, a partir de su participación en un proyecto global de Open Society Foundations en 2011; así como "Overcoming citizen mistrust and enhancing democratic practices: Results from the e-participation platform México Participa” de María Elena Meneses Rocha y Alejandro Martín del Campo, del Tecnológico de Monterrey con colegas de la University of California Berkeley.

Por otro lado, la búsqueda de la academia por incidir en los debates públicos quedó registrada en "Un ejercicio de memoria para entender las reformas en telecomunicaciones y radiodifusión. La participación de la sociedad civil y la academia”, de Fátima Fernández Christlieb, Patricia Ortega Ramírez y Beatriz Solís Leree.

En suma, esta revisión cronológica permite identificar etapas en los estudios sobre internet, comunicación y cultura digital, cuyos avances y hallazgos se presentaron en las publicaciones analizadas. Como se aprecia en esta sección, la década de 1980 concentró las preocupaciones iniciales ante la llegada de las TIC y sus implicaciones en diferentes campos de la vida social. En la década de 1990 se situaron los primeros abordajes sobre internet y se registró un desplazamiento del enfoque en las posibilidades, o bien en las descripciones, para avanzar hacia el estudio de los usos y las prácticas de comunicación en entornos digitales. En la década del 2000 hay importantes aportaciones teóricas y metodológicas, que permitieron encuadrar los intereses y las preguntas, a la vez que crecieron las investigaciones empíricas. Esta última tendencia se mantuvo y creció en la década del 2010, al tiempo que se diversificaron las temáticas de cruce con lo digital.

Las tradiciones del pensamiento comunicacional latinoamericano se identifican con claridad, sobre todo en los textos de los primeros años. La tradición crítica estuvo presente desde el inicio de los trabajos. De hecho, Héctor Schmucler - que es una de las figuras clave de ésta - abrió 
tempranamente la discusión sobre los desafíos que traerían las TIC a la investigación sobre comunicación, desde 1981. Otras contribuciones en esta línea son las de Javier Esteinou Madrid, Fátima Fernández Christlieb y Florence Toussaint, entre otros.

La tradición culturalista se identifica en trabajos que llegaron después. Se trata, además, de un cuerpo de trabajos y de académicos que se ha ido expandiendo a lo largo de los años. Como se mencionó anteriormente, dos figuras clave de esta tradición han sido Jesús Martín-Barbero y Néstor García Canclini. El primero vivió algunos años en México y el segundo ha tenido una estancia prolongada y productiva en este país. Algunas de las contribuciones teóricas importantes son obra de ellos. En este grupo también puede identificarse el trabajo de investigadores de la línea sociocultural, tales como Raúl Fuentes Navarro, Carmen Gómez Mont y otros.

La tradición alternativista, por su naturaleza, no siempre es evidente en los productos académicos, ya que su énfasis suele estar en la intervención social. Si bien hay casos conocidos e interesantes en México, de proyectos comunitarios en medios y gestión cultural, son minoría los que aparecieron en esta revisión. En esa línea se puede ubicar el trabajo de Ana Isabel Zermeño Flores y su equipo en la Universidad de Colima, que han integrado la intervención mediante proyectos de comunicación para el cambio social y la publicación.

Más allá de las tradiciones del pensamiento comunicacional latinoamericano identificadas por Kaplún (2013), es posible identificar dos tradiciones más entre los trabajos abordados en esta revisión. Por un lado, se aprecia la herencia de Marshall McLuhan y la perspectiva de la ecología mediática en textos de investigadores como Octavio Islas, Fernando Gutiérrez, Claudia Benassini, entre otros. Por otro lado, se observan los enfoques de la complejidad, que hacen autores como Jorge González, Margarita Maass Moreno y José Amozurrutia.

Sin embargo, así como en muchos trabajos se identifican con claridad los posicionamientos, en buena parte de los estudios - sobre todo en los más recientes - hay una ausencia de esto. Como ha planteado 
Fuentes Navarro (2011), hay una fragmentación del campo. Además, si bien se requiere mayor análisis, se observa que en los primeros años había poco trabajo empírico, mucho trabajo ensayístico, aunque las posiciones solían ser más claras. Con el paso de los años, hay más trabajo empírico, pero, en algunos casos, éste se limita a la presentación de los resultados, sin mayor discusión de la teoría y a veces sin un posicionamiento frente a lo que se estudia.

\section{Conclusiones}

En este artículo se presentó una revisión de publicaciones sobre internet, comunicación y cultura digital, en México, realizada a partir de libros, capítulos en libros y artículos en revistas especializadas, publicados entre 1981 y 2019. En la lógica del pensamiento comunicacional (MIÈGE, 1995), se identifican etapas en estos trabajos. Si al pensamiento comunicacional se le agrega el apellido latinoamericano es posible identificar rasgos de al menos tres de las cuatro tradiciones que plantea Kaplún (2013): la crítica, la culturalista y, en cierta medida, la alternativista. Asimismo, se identifica la presencia del pensamiento mcluhaniano y de la complejidad en algunas otras aportaciones.

Como varios autores han señalado, hay una relativa ausencia de teorización, de desarrollo metodológico, así como de perspectivas regionales y comparativas, que se ha mantenido a lo largo del tiempo (FUENTES NAVARRO, 2000, 2011; GÓMEZ-CRUZ \& SILES, 2020; SCOLARI, 2008; SILES, 2008; SILES, ESPINOZA ROJAS, \& MÉNDEZ MARENCO , 2019; TREJO DELARBRE, 1996, 2009, 2013).

La presente revisión tuvo como base un análisis de contenido que se leyó en clave de línea de tiempo para identificar temáticas emergentes y autores clave. Se observa que el desarrollo temático ha ido de preocupaciones generales sobre las posibilidades de las inicialmente llamadas nuevas tecnologías hacia preocupaciones específicas por el vínculo entre medios digitales y educación, política, juventud, movimientos sociales, medios de comunicación, entre otros. Algunas de estas temáticas se relacionan con el contexto sociopolítico nacional, como los 
estudios de movimientos sociales, en torno a los zapatistas, \#YoSoyl32 o Ayotzinapa; o bien a la innovación en plataformas y aplicaciones, como muestran las investigaciones sobre el uso de blogs, Facebook, Twitter o YouTube.

Las y los autores que fueron mencionados en el cuerpo del texto son - casi en todos los casos - quienes más veces aparecen en los productos registrados. Más allá de la cantidad, se trata de investigadores que han hecho aportaciones teóricas y/o metodológicas para la investigación sobre internet, comunicación y cultura digital, que es importante reconocer y discutir.

A reserva de realizar un análisis más profundo de las temáticas específicas y las perspectivas teóricas y metodológicas desde las cuales se han abordado los objetos, esta revisión contribuye a reconocer las aportaciones de las y los académicos mexicanos en el marco del pensamiento comunicacional latinoamericano.

Como se ha visto, algunos de los referentes clave del pensamiento comunicacional latinoamericano que identificó Kaplún (2013) pasaron algunos años o se establecieron definitivamente en México - tal fue el caso de Héctor Schmucler, Jesús Martín-Barbero y Néstor García Canclini- y eso ha permeado en cierta medida las tradiciones de estudio a lo largo de estos años. Sin embargo, la fragmentación que señalaba Fuentes Navarro (2011) dificulta las identificación de estas líneas en las décadas más recientes.

Las observaciones de Gómez Cruz y Siles (2020) sobre el estudio de la cultura digital en Latinoamérica aplican también para esta revisión de publicaciones en México. Son problemáticas que se han arrastrado durante varios años (FUENTES NAVARRO, 2000, 2011; SCOLARI, 2008; SILES, 2008; SILES, ESPINOZA ROJAS, \& MÉNDEZ MARENCO , 2019; TREJO DELARBRE, 1996, 2009, 2013). Hay una ausencia de teorización que abre otras problemáticas, como la ausencia de bases comunes para discutir objetos, conceptos y métodos; las tendencias a confirmar ideas establecidas en lugar de explicar nuevos 
fenómenos, a la especulación teórica más que a la investigación empírica, así como a emplear métodos tradicionales más que a innovar.

En el caso mexicano, si bien las publicaciones muestran un aumento de trabajos empíricos, esto no tiene su correlato en la producción teórica y la innovación metodológica. Además, aunque la emergencia de temáticas vinculadas al contexto sociopolítico y/o a la innovación en plataformas y aplicaciones, puede entenderse dentro de la lógica latinoamericana de compromiso con las problemáticas nacionales y regionales, estamos asumiendo posiciones más reactivas que propositivas, lo que nos coloca del lado del riesgo que señaló Schmucler (1981) hace casi cuatro décadas: "anticiparse a los acontecimientos y no resignarse a estudiar, retroactivamente, los efectos de los nuevos sistemas comunicativos" (p. 61).

Esto se traduce como un desafío para distintas generaciones de investigadores, principalmente las más jóvenes, de reconocer las raíces del trabajo realizado y contribuir a solventar las asignaturas que por años han quedado pendientes. No es un dato menor que algunas de las aportaciones teóricas más relevantes sean de hace más de 15 años. La pregunta que queda abierta es cómo y de qué manera construiremos nuestro futuro en el campo de la comunicación y, específicamente, en el estudio de internet, la comunicación y la cultura digital.

\section{Referencias}

ENGHEL, F., \& BECERRA, M. Here and there: (Re)situating Latin America in international communication theory. Washington, v. 28, n. 2, p. 111-130, 2018.

FUENTES NAVARRO, R. Educación y telemática. Bogotá: Grupo Editorial Norma, 2000.

FUENTES NAVARRO, R. Exploraciones teórico-metodológicas para la investigación sociocultural de los usos de la Internet. En VASSALLO DE LOPES M.I. \& FUENTES NAVARRO R. (Coord.). Comunicación, campo y objeto de estudio. Perspectivas reflexivas latinoamericanas. Guadalajara: ITESO / Universidad Autónoma de Aguascalientes / Universidad de Colima / Universidad de Guadalajara, 2001, p. 229-245.

FUENTES NAVARRO, R. Pensamiento comunicacional latinoamericano y convergencia digital. Retos epistemológicos y académicos. En DEL VALLE C., MORENO F. \& 
SIERRA F. (Coord.). Cultura latina y revolución digital. Matrices para pensar el espacio iberoamericano de comunicación. Barcelona: Gedisa, 2011, p. 41-68.

FUENTES NAVARRO, R. Recursos para la desfragmentación de la investigación iberoamericana en comunicación. São Paulo, n. 20, p. 16-25, 2014.

GANTHER, S., \& ORTEGA, F. The invisibility of Latin American scholarship in European media and communication studies: Challenges and opportunities of de-Westernization and academic cosmopolitanism. Los Angeles, v. 13, p. 68-91, 2019.

GÓMEZ-CRUZ, E., \& SILES, I. Digital cultures in the Americas. En RAUSSERT W., ANATOL G., THIES S., CORONA S. \& LOZANO J.C. (Ed). The Routledge handbook to the cultures and media of the Americas. London: Routledge, 2020, p. 319-329.

KAPLÚN, G. Viejas y nuevas tradiciones en la comunicación latinoamericana. São Paulo, v. 10, n. 18, p. 66-76, 2013.

KRIPPENDORFF, K. Content analysis. London: SAGE, 2004.

MARQUES DE MELO, J. Los derechos humanos como pilares éticos en el legado comunicacional de Paulo Freire y Luiz Beltrão. Cochabamba, n. 23, p. 9-17, 2011.

MENESES, M., \& PÉREZ SALAZAR, G. Cómo y qué se estudia sobre Internet y Sociedad de la Información en México. Una mirada desde la AMIC. Guadalajara, n. 26, p. 43-70, 2016.

MIÈGE, B. Las etapas del pensamiento comunicacional. Bogotá, n. 26, p. 109-138. 1995.

SCHMUCLER, H. La hora de las computadoras. El estudio de la comunicación en América Latina. México, n. 1, p. 47-62, 1981.

SCOLARI, C. Hipermediaciones. Elementos para una teoría de la comunicación digital interactiva. Barcelona: Gedisa, 2008.

SILES, I. A la conquista del mundo en línea: internet como objeto de estudio (19902007). Guadalajara, n. 10, p. 55-79, 2008.

SILES, I., ESPINOZA ROJAS, J., \& MÉNDEZ MARENCO , A. La investigación sobre tecnología de la comunicación en América Latina: un análisis crítico de la literatura (2005-2015). Bogotá, v. 22, n. 1., p. 12-40, 2019.

TREJO DELARBRE, R. La nueva alfombra mágica. Usos y mitos de Internet, la red de redes. Madrid / Ciudad de México: FUNDESCO / Editorial Diana, 1996.

TREJO DELARBRE, R. La investigación latinoamericana sobre Internet. Brechas digitales y diversidad analítica. Madrid, n. 61, p. 1-8, 2009.

TREJO DELARBRE, R. The Study of the Internet in Latin America: achievements, challenges, futures. En VALDIVIA A. (Ed.). The International Encyclopedia of Media Studies. NJ: Blackwell, 2013, p. 1-29.

TREJO DELARBRE, R., \& SOSA PLATA, G. Campo nuevo, problemas viejos. La investigación mexicana en materia de Sociedad de la Información, Internet, Cibercultura y Telecomunicaciónes. En VEGA MONTIEL, A. La comunicación en México. Una agenda de investigación. Ciudad de México: UNAM / AMIC, 2009, p. 1-23. 


\section{Sobre la autora:}

Dorismilda Flores-Márquez - Profesora investigadora en la Facultad de Comunicación y Mercadotecnia de la Universidad De La Salle Bajío.

Data de submissão: 12/09/2020

Data de aceite: 26/10/2020 